\title{
Overactive bladder (OAB) in women: questionnaire based approach
}

\author{
Veena Ganju Malla, AmitaTuteja, Pushpa Singh \\ Corresponding author: Dr Veena Ganju Malla, Professor, Department of Obstetrics and \\ Gynaecology, ABVIMS \& Dr. RML Hospital, New Delhi, India; Email - veenaganju@yahoo.com
}

Distributed under Attribution-Non Commercial - Share Alike 4.0 International (CC BY-NC-SA 4.0)

\begin{abstract}
Objectives: We aim to study the prevalence of various lower urinary tract symptoms in women with overactive bladder (OAB). We also evaluated the impact of OAB on patient's quality of life (QOL) through various validated self reporting questionnaires. Methods: An observational cross-sectional study was done where a total of 200 patients who were confirmed cases of $\mathrm{OAB}$ were included in the study. The patients were questioned using four structured well designed validated questionnaires and the data were recorded and analysed. Results: Age of patients ranged from 18 to 72 years. Urine frequency (99\%) was the most commonly reported symptom followed by nocturia (91\%) and urgency (89\%). The mean scores for American urology association symptom index (AUASI) was 17, total bothersomeness score (TBS):30.17, urogenital distress inventory (UDI) - 6:58.75 and incontinence impact questionnaire (IIQ -7) was 75.95. Higher scores in IIQ-7 and UDI-6 correlated well with negative impact on quality of life and this association was found to be statistically significant. Conclusion: This study offers an introduction to the concept of QOL assessment using short form questionnaires for patient with symptoms of OAB. Many sufferers seek to manage their symptoms by adjusting to the problem instead of seeking treatment. This has not only puts a negative impact on the quality of life of women but also puts a financial burden on society especially in the developing countries.
\end{abstract}

Keywords: Overactive bladder, questionnaires, quality of life.

Overactive bladder $(\mathrm{OAB})$ is defined as "urinary urgency, usually with frequency and nocturia, with or without urgency urinary incontinence (UUI), in the absence of urinary tract infection (UTI) or other obvious pathology". The condition is highly prevalent and is associated with significant economic burden and lower health related quality of life (HRQoL). It affects millions of people worldwide but often goes unreported as most women consider it as part of normal ageing and suffer in silence. Even the previous studies have focussed on incontinence and therefore prevalence and clinical impact of $\mathrm{OAB}$ has been grossly underestimated and thus not managed properly.

Although $\mathrm{OAB}$ is not life threatening, but symptoms associated with it can have a profound impact on quality of life of women. Despite the negative impact of $\mathrm{OAB}$ on HRQoL, a recent registry-based, online survey study which was conducted across various countries found that a major proportion of such patients never consult a physician regarding their urine related bladder symptoms. In addition, the study also found that the ones who consulted waited for long period before the symptoms were so troublesome that they could not avoid consultation.

Received: $10^{\text {th }}$ March 2020. Accepted: $14^{\text {th }}$ April 2020.

Malla VG, Tuteja A, Singh P. Overactive bladder (OAB) in women: questionnaire based approach. The New Indian Journal of OBGYN. 2020; 7(1): 26-30. 
Above all, the physicians assessment of the disease burden of these symptoms is shown to be inaccurate and non reproducible. Thus, considering the high prevalence of $\mathrm{OAB}$ in women, in conjunction with the fact that many patients fail to mention their problems during clinical consultations, women may benefit from screening for symptoms related to $\mathrm{OAB}$, including UUI, through self reporting questionnaires.

The advent of validated condition specific questionnaires proves to be a rapid technique of eliciting the impact of symptoms on quality of life and has encouraged their inclusion in clinical trials of continence care. Only by fully understanding the impact of urinary incontinence on quality of life of women can hope to improve its treatment. Here, we aim to study the prevalence of various lower urinary tract symptoms in women with $\mathrm{OAB}$. We also evaluated the impact of OAB on patient's quality of life (QOL) through various validated self reporting questionnaires.

\section{Materials and methods}

An observational cross-sectional study was done where a total of 200 patients who were confirmed cases of OAB according to the ICS definition1 and attending urogynaecology clinic who agreed to participate in the study were included in the study. The patients were questioned using four structured well designed validated questionnaires in detail to enquire about their symptoms and its impact on quality of life. Informed consent was taken from them. Institutional ethical committee approval was taken for the study. The patients were asked questions about urinary tract symptoms, demographic data (age, marital status, parity), BMI, menopausal status, history of surgical intervention and any associated comorbidities. The diagnosis of $\mathrm{OAB}$ in the present study was based on self reported symptoms according to the ICS definition. 1

To obtain an OAB specific QOL symptoms assessment, AUASI (American urology association symptom index), and total bothersomeness score (TBS) were used and for measuring the impact of the symptoms on the QOL, short forms of urogenital distress inventory (UDI-6)4 and incontinence impact questionnaire (IIQ-7) were used. AUASI questionnaire consisted of 7 items, each with a 5 point response scale. The UDI-6 questionnaire had 6 items each with a 3 point response scale, which were summed and transformed to a 0 to 75 scale. The short form IIQ-7 questionnaire consists of 7 items, covering 4 domains; physical activity, social relationships, travel and emotional health, each with a 4 point response scale. Patients were asked to rate the extent to which their urinary symptoms affect their daily functioning from 0 (not at all) to 3(greatly). The items scored were summed and then transformed to a 0 to 100 scale. A high score indicated worse quality of life in both UDI--6 and IIQ-7 questionnaires. Data was coded and analysed using descriptive statistics, which were reported as the mean and standard deviation (quantitative variables) or as the number and percentage (qualitative variables). The statistical analysis was carried out by calculating the Pearsons correlation coefficient. A 5\% level of significance was used throughout the present study.

\section{Results}

Patients ranged from 18 to 72 years with majority being in the 36 to $50 \mathrm{yr}$ age group. $63 \%$ patients were with parity

Table 1: Demographic characteristics

\begin{tabular}{lll}
\hline Categories & & Percentages \\
\hline Age in years & $18-35$ & 28 \\
& $36-50$ & 46 \\
Parity & $>50$ & 26 \\
& $<2$ & 37 \\
BMI $\left(\mathrm{Kg} / \mathrm{m}^{2}\right)$ & $>3$ & 63 \\
& $<19$ & 7 \\
& $19-23$ & 24 \\
H/O Hysterectomy & $23.1-27$ & 35 \\
& $>27$ & 34 \\
Postmenopausal women & Present & 20 \\
Comorbidities & Absent & 80 \\
& & 49 \\
& Present & 57 \\
\hline
\end{tabular}

more than 3, 69\% with BMI of $23,49 \%$ of women were postmenopausal with history of hysterectomy in $20 \%$ of patients. The incidence of associated medical comorbidities was $57 \%$ (table-1). In our study, symptoms attributable to $\mathrm{OAB}$ were identified by responses to questions on frequency, urgency and urinary incontinence. Frequency (99\%) was the

Table 2: Urinary Symptoms among patients

\begin{tabular}{ll}
\hline Symptoms & Percentage \\
\hline Frequency & 99 \\
Nocturia & 91 \\
Urgency & 89 \\
Incomplete Evacuation & 67 \\
Dysuria & 45 \\
\hline most commonly reported symptom followed by nocturia
\end{tabular}
$(91 \%)$ and urgency $(89 \%)$. An overlap of symptoms was seen in most of the patients (table - 2). The vast majority of patients had a symptom duration of 39 months with mean duration of $43.29 \pm 26.38$ months. Frequency, nocturia and urgency was found to be the most bothersome (figure -1). The mean scores for AUASI was 17, TBS: 30.17, UDI- 
6:58.75 and IIQ -7 was 75.95. Higher scores in IIQ-7 and UDI-6 correlated well with negative impact on quality of life

Table 3: Correlation of Severity of symptoms with quality of life

\begin{tabular}{lll}
\hline $\begin{array}{l}\text { Scores (Pearson } \\
\text { Correlation) }\end{array}$ & $\begin{array}{l}\text { UDI-6 } \\
\text { (r value) }\end{array}$ & $\begin{array}{l}\text { IIQ-7 } \\
\text { (r-value) }\end{array}$ \\
\hline AUASI & 0.212 & 0.234 \\
TBS & 0.328 & 0.584 \\
\hline
\end{tabular}

and this association was found to be statistically significant (table-3). Higher the AUASI and TBS scores, more was the

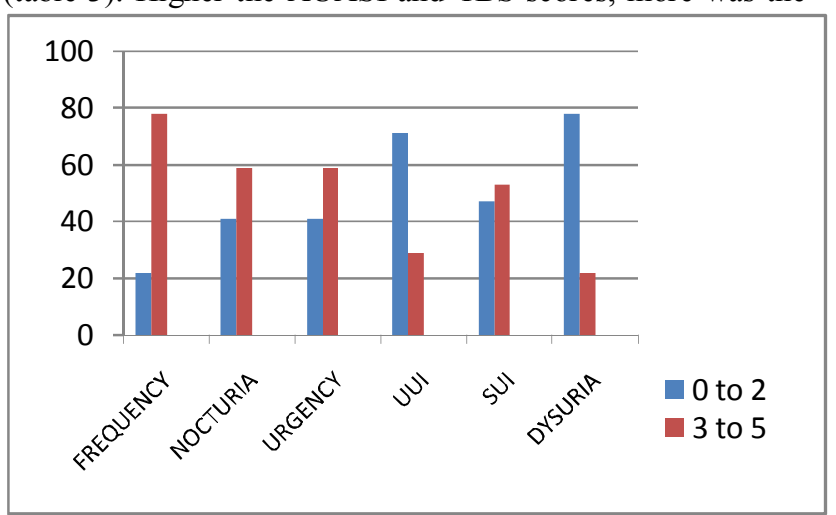

Figure 1: Degree of bothersome caused by each individual symptom (using Likert scale 0-2 versus 3-5).

Table 4: Patients satisfaction with the current symptomatology

\begin{tabular}{ll}
\hline Parameter & Percentage \\
\hline Satisfied & 5 \\
Mixed & 21 \\
Mostly dis-satisfied & $39 \%$ \\
Unhappy & 24 \\
Terrible & 11 \\
\hline
\end{tabular}

quality of life affected. In our study, $39 \%$ of patients were "mostly dissatisfied - 4 points" if they were to spend the rest of their lives with the urinary problem (table - 4).

\section{Discussion}

The observations revealed that magnitude of lower urinary tract symptoms increased with age and $72 \%$ of women were above $35 \mathrm{yrs}$ of age. Several other studies confirm the findings that $\mathrm{OAB}$ symptoms increase linearly with advancing age. High parity $(>3)$, obesity, menopause, presence of comorbidities were seen as associated findings in women with $\mathrm{OAB}$. The findings are in unison with current evidence that increasing age, high BMI, parity and associated medical comorbidity are risk factors associated with lower urinary tract symptoms.

In our study the prevalence of OAB in patients who had undergone hysterectomy was $20 \%$. While several studies have shown higher rates of LUTS in women who underwent hysterectomy others have not confirmed this correlation.

It is known that $\mathrm{OAB}$ cause embarrassment, reduce self esteem and lead to impaired emotional and psychological well being. Although incontinence is highly prevalent and bothersome condition, less than half of community dwelling adult women with symptoms have talked to a physician about urinary problem. Some of the factors associated with treatment seeking were symptoms for more than 36 months duration, having a history of noticeable accidents, with worse QOL scores. In our study, the median duration of symptoms after which the patient first presented was 39 months. Milsom et al also highlight that $79 \%$ of patients had symptoms for more than 1 year and $49 \%$ for more than 3 years.9 It clearly highlights that LUTS are not perceived as a problem that warrants medical help. General practitioners should raise the awareness as a part of routine general health checkups.

In our study, frequency and nocturia were the commonest LUTS in women of overactive bladder, present in $99 \%$ and $91 \%$ respectively. The study depicts that OAB with and without incontinence influences quality of life in a negative way. The statistically significant correlation of high AUASI and TBS scores with high UDI-6 and IIQ-7 scores clearly emphasizes the negative impact of lower urinary tract symptoms on a woman's life.

Quality of life impact is an essential component of the patient centred paradigm and validated assessment tools show good correlation. The American Urological Association Symptom Index (AUASI) is a series of seven questions that assess symptoms of storage and voiding. Though its original use was in benign prostatic hypertrophy in men, its use in female patients has been investigated but not well established. Scarpero et al. looked at the use of the AUASI and whether it correlates in women with the level of bother caused by lower urinary tract symptoms. They also determined whether the association varies with a woman's age and continent status and whether the AUASI and degree of bother correlate with the patient's quality of life assessment. The charts of over 1200 women were reviewed who filled out the AUASI as well as quality of life measurement. There was a strong correlation (defined as correlation coefficient value greater than 0.8 , with maximum of 1.0) between symptoms and problems caused by symptoms $(0.858, \mathrm{P}<0.0001)$. The correlation existed throughout various age groups and was independent of 
The New Indian Journal of OBGYN. 2020 (July-December); 7(1)

coexisting incontinence. The symptoms correlated with quality of life in a similar pattern.

UDI-6 and IIQ-7 taken together assess urinary symptoms, the symptom bother, and the impact the bother has on patients' quality of life. In clinical application, it may be of significant use and help us treat the patients in an effective way. Other questionnaires include The Bristol Female Lower Urinary Tract Symptoms (BFLUTS) Questionnaire which was also developed with an emphasis on symptom quantification as well as on quality of life. In the post-survey psychometric assessment, the questionnaire demonstrated reliability and validity. Construct validity and criterion validity were demonstrated. Reliability was good; a Cronbach's alpha of 0.78 indicated that the symptom questions had high internal consistency, while stability was excellent, with $78 \%$ of symptoms and problems answered identically on two occasions, and strong (greater than 0.8) Spearman rank correlation coefficients of 0.86 and 0.90 , respectively. The OAB-q questionnaire which was designed to assess symptom bother and quality of life in patients with both continent and incontinent $\mathrm{OAB}$; the Urgency Questionnaire (UQ) which consists of 15 Likert-scale items and four visual analogue scales rating the severity and impact of urinary urgency symptoms; the Primary OAB Symptom Questionnaire (POSQ) which is a five-item questionnaire that assesses which symptom of $\mathrm{OAB}$ is the most bothersome to patients; the Overactive Bladder Satisfaction Questionnaire (OAB-S) which was developed to assess patients' satisfaction with $\mathrm{OAB}$ treatment including medications or other treatments, i.e., physical therapy or biofeedback; the International Consultation on Incontinence Questionnaire (ICIQ) which comprise of three scored items (prevalence, frequency, and impact on quality of life) and an unscored self-diagnostic item (perceived cause of leak) and which is now modified in to ICIQ Modular Questionnaire.

The use of these self reporting questionnaires can better help the patients to assess themselves individually by themselves as done in breast examination by females at home. Awareness and regional questionnaires forms needs to be created to ameliorate LUTS which has been growing among women and is a cause of concern and discomfort among the mid-life.

\section{Conclusion}

Questionnaires are important tools for assessing the prevalence and impact of OAB on quality of life of sufferers. Many sufferers seek to manage their symptoms by adjusting to the problem instead of seeking treatment. This has not only put a negative impact on the quality of life of women but also puts a financial burden on society. Often the severity of the problem and impact of treatment are measured by urodynamic studies which are cumbersome, costly and fail to measure bothersomeness and effect on patient's life. This study offers an introduction to the concept of QOL assessment using short form questionnaires for patient with symptoms of $\mathrm{OAB}$.

\section{Conflict of interest: None. Disclaimer: Nil.}

\section{References}

1. Haylen BT, de Ridder D, Freeman RM, Swift SE, Berghmans $\mathrm{B}$, Lee $\mathrm{J}$, et al. An International Urogynecological Association (IUGA)/International Continence Society (ICS) joint report on the terminology for female pelvic floor dysfunction. Int Urogynecol J. 2010; 21(1): 5-26.

2. Cardozo L, Staskin D, Currie B, Wiklund I, Globe D, Signori M, et al. Validation of a bladder symptom screening tool in women with incontinence due to overactive bladder. Int Urogynecol J. 2014; 25(12):1655-63.

3. Cruz F, Denys P, Cidre MJ, Signori M, Globe D. Patient attitudes and patterns of treatment utilization in a European population with overactive bladder. Poster presented at the European Association of Urology (EAU) 2012 27th Annual Congress. 2011: Paris, France; 24-8.

4. Svatek R, Roche V, Thornberg J, Zimmern P. Normative values for the American Urological Association Symptom Index (AUA-7) and short form Urogenital Distress Inventory (UDI-6) in patients 65 and older presenting for non-urological care. Neurourol Urodyn. 2005; 24(7): 606-10.

5. van de Vaart H, Falconer C, Quail D, Timlin L, Manning M, Tincello D, et al. Patient reported outcomes tools in an observational study of female stress urinary incontinence. Neurourol Urodyn. 2010 Mar; 29(3): 348-53.

6. Botlero R. Age specific prevalence of and factors associated with different types of urinary incontinence in Community dwelling Australian women assessed with a validated questionnaire. Maturitas. 2009 Feb 20; 62(2): 134-9.

7. Grodstein F. Association of age, race and obstetric history with urinary symptoms among women in the 
The New Indian Journal of OBGYN. 2020 (July-December); 7(1)

nurses health study. Am J Obstet Gynaecol. 2003: 189: 428-34.

8. Milsom I. The influence of age, parity, oral contraception, hysterectomy and menopause on the prevalence of urinary incontinence in women. J Urol. 1993; 149: 1459-62.

9. Samuelsson E. Determinants of urinary incontinence in a population of young and middle aged women. Acta Obstet Gynaecol Scand. 2000; 79: 208-15.

10. Scarpero HM, Fiske J, Xue X, Nitti VW. American Urological Association Symptom Index for lower urinary tract symptoms in women: correlation with degree of bother and impact on quality of life. Urology. 2003; 61: 1118-22.

11. Jackson S, Donovan J, Brookes S, Eckford S, Swithinbank L, Abrams P. The Bristol Female Lower Urinary Tract Symptoms Questionnaires: development and psychometric testing. Br J Urol. 1996; 7: 805-12.

12. Shy M, Fletcher SG. Objective Evaluation of Overactive Bladder: Which Surveys Should I Use? Curr Bladder Dysfunct Rep. 2013 Mar 1; 8(1): 45-50.

Veena Ganju Malla ${ }^{1}$, AmitaTuteja $^{2}$, Pushpa Singh ${ }^{3}$

$1,2,3$ Department of Obstetrics and Gynaecology, ABVIMS \& Dr. RML Hospital, New Delhi, India 